\title{
Effect of whole body vibration in Parkinson's disease: A controlled study
}

\author{
Pablo Arias, PhD, Marcelo Chouza, BSc, Jamile Vivas, PhD, and Javier Cudeiro, MD, \\ $\mathrm{PhD}$
}

\begin{abstract}
In the search of new strategies to improve the quality of life of Parkinson's disease patients, recent work has reported an amelioration of Parkinsonian symptoms using Whole Body Vibration (WBV). A double-blinded, placebo controlled design was used to evaluate the effect of a 12 WBV sessions-programme on a number of motor and clinical tests in 23 Parkinson's disease patients. Patients were assigned to one of two groups, one receiving WBV and the other a placebo group. At the end of the programme as well as during intra-session evaluation, there was no difference between the experimental (vibration) and placebo groups in any outcomes. These results suggest that reported benefits of vibration are due to a placebo response.
\end{abstract}

The use of vibrating platforms has been introduced in the sports medicine aiming to improve strength in disciplines requiring powerful muscle contractions, though its effectiveness has not yet been clearly demonstrated. ${ }^{1}$ Recently, this technique has been used in Parkinson's disease patients, with some promising results. ${ }^{2-4}$ Haas et al. ${ }^{3}$ have reported an improvement UPDRS scores (rigidity and tremor) in a group of patients after undergoing a single session of whole body vibration (WBV). Previous reports by the same group, ${ }^{2}$ showed a significant improvement in postural stability on an unstable platform after one session of WBV. Both studies were performed by applying five sets of 60 seconds each, at $6 \mathrm{~Hz}$, employing stochastic vibration which was one of the key features advocated by the authors. ${ }^{5}$ However, more recently, significant effects on Parkinsonian patients have been reported using nonstochastic vibration. ${ }^{4}$ These authors showed the effect of $25 \mathrm{~Hz}$ stimulation (twice per day, $15 \mathrm{~min}$./each) for 3 weeks which produced an improvement in a set of motor tests (Tinetti balance score, ${ }^{6}$ gait velocity, standwalk-sit test, UPDRS ${ }_{\text {III }}$ ), although similar results were seen with conventional rehabilitation therapy (relaxation, occupational therapy, etc.). However, the results by Ebersbach et al. ${ }^{4}$ seem important, since, besides the acute effects reported previously, ${ }^{2},{ }^{3}$ they appeared to show long lasting effects. Improvement after the end of the 3 week programme lasted at least 4 more weeks. Nevertheless, as stated by the authors, ${ }^{2-4}$ the lack of a control (placebo) is a weakness of these studies. The effect of placebo on different pathologic conditions is well know, and it seems Parkinsonian patients are especially affected, ${ }^{7,8}$ chiefly when a reward is expected (improvement in clinical symptoms due to a new treatment, for instance).

This study aims to evaluate the effect of WBV on motor symptoms on Parkinson's disease patients, while controlling for the placebo effect.

\section{METHODS}

\section{Patients}

A total of 29 Parkinson's disease patients, belonging to Asociación Parkinson Bueu in Spain were recruited for the study. To take part in the study, patients had to match the primary diagnosis of Parkinson's disease (PD) (based on medical records following established diagnostic criteria); lack of dementia (MMSE $\geq 24$ ); lack of artromuscular deficit or joint prosthesis; and be able to cope with OFF periods $(n=23)$. Patients were required to complete all sessions and could not change their regime of medication throughout the study. Patients were allocated to either experimental or placebo group based upon an ABBA ( $\mathrm{A}=$ =xperimental; $\mathrm{B}=$ =placebo) distribution model, using their times to complete the Gait test. Although initial allocations placed 12 patients in the experimental group and 11 in the placebo group, 
which were not significantly different in any pretest measure, 2 patients withdrew from the study. Pretest data for the remaining 10 experimental and 11 placebo patients were still not significantly different $(\mathrm{P}>$ 0.05), neither their weight, height, trochanteral height, and acromion-finger length (Table 1).

TABLE 1. Subjects

\begin{tabular}{|c|c|c|c|c|c|c|}
\hline Patient (Group) & Sex & Age & Weight (kg) & Height (m) & Arm length (m) & Trochanteral height (m) \\
\hline 1(Placebo) & $\mathrm{F}$ & 65 & 66 & 1.55 & 0.73 & 0.75 \\
\hline 2(Placebo) & M & 75 & 64 & 1.59 & 0.77 & 0.79 \\
\hline 3(Placebo) & $\mathrm{F}$ & 58 & 87 & 1.57 & 0.77 & 0.83 \\
\hline 4(Placebo) & $\mathrm{F}$ & 73 & 68 & 1.60 & 0.76 & 0.78 \\
\hline 5(Placebo) & M & 59 & 81 & 1.67 & 0.74 & 0.80 \\
\hline 6(Placebo) & M & 72 & 87 & 1.80 & 0.81 & 0.85 \\
\hline 7(Placebo) & M & 67 & 75 & 1.70 & 0.78 & 0.85 \\
\hline 8(Placebo) & $\mathrm{F}$ & 65 & 75 & 1.65 & 0.75 & 0.81 \\
\hline 9(Placebo) & M & 66 & 74 & 1.73 & 0.79 & 0.83 \\
\hline 10(Placebo) & $\mathrm{F}$ & 62 & 78 & 1.70 & 0.77 & 0.83 \\
\hline 11(Placebo) & M & 70 & 70 & 1.67 & 0.79 & 0.83 \\
\hline Group Mean & & 66.55 & 75.00 & 1.66 & 0.77 & 0.81 \\
\hline$( \pm s d)$ & & $( \pm 5.57)$ & $( \pm 7.81)$ & $( \pm 0.08)$ & $( \pm 0.02)$ & $( \pm 0.03)$ \\
\hline 1(Experimental) & $\mathrm{F}$ & 67 & 68 & 1.47 & 0.65 & 0.74 \\
\hline 2(Experimental) & M & 79 & 72 & 1.61 & 0.71 & 0.84 \\
\hline 3(Experimental) & $\mathrm{F}$ & 74 & 86 & 1.62 & 0.72 & 0.84 \\
\hline 4(Experimental) & $\mathrm{F}$ & 49 & 75 & 1.53 & 0.71 & 0.83 \\
\hline 5(Experimental) & M & 78 & 68 & 1.61 & 0.71 & 0.83 \\
\hline 6(Experimental) & M & 79 & 71 & 1.63 & 0.74 & 0.84 \\
\hline 7(Experimental) & $\mathrm{M}$ & 62 & 79 & 1.71 & 0.82 & 0.85 \\
\hline 8(Experimental) & M & 55 & 67 & 1.66 & 0.78 & 0.85 \\
\hline 9(Experimental) & $\mathrm{F}$ & 55 & 63 & 1.50 & 0.71 & 0.81 \\
\hline 10(Experimental) & $\mathrm{M}$ & 71 & 83 & 1.72 & 0.84 & 0.91 \\
\hline Group Mean & & 66.90 & 73.20 & 1.61 & 0.74 & 0.83 \\
\hline$( \pm s d)$ & & $( \pm 11.11)$ & $( \pm 7.45)$ & $( \pm 0.08)$ & $( \pm 0.06)$ & $( \pm 0.04)$ \\
\hline
\end{tabular}

\section{Procedure}

\section{Stimulation}

The experimental group received 12 stimulation sessions over 5 weeks, on nonconsecutive days. Based on previous studies reporting beneficial effects on Parkinsonian symptoms ${ }^{2,3}$ the stimulation frequency was set at $6 \mathrm{~Hz}$., and each patient underwent 5 sets of stimulation of 1 min each, with an interset rest period of $1 \mathrm{~min}$. During stimulation patients were asked to stand on the vibrating platform, with their feet separated at a stable and comfortable position, and with the knees slightly bent.

For the placebo condition patients adopted the same position but vibration was not applied. Instead placebo patients were required to try to stay as still as they could, trying to minimize hip oscillation, yielding the same amount of weight of both feet.

All other physical therapies usually undergone by the patients were cancelled during the duration of this study. Patients took their medication as usual, and stimulation session started 30-45 min after dose intake (when patients confirmed ON-periods).

\section{Evaluation}

We assessed the effect of vibration through two different sets of tests, one to test the effectiveness of the whole programme, and other measuring the acute effect of the different sessions (intra-session evaluation), see Table 2. 
TABLE 2. Evaluation protocol

\begin{tabular}{|c|c|c|}
\hline Preprogramme OFF-periods & $\begin{array}{l}\text { Stimulation programme (sessions) ON- } \\
\text { periods }\end{array}$ & Postprogramme OFF-periods \\
\hline \multirow[t]{2}{*}{$\begin{array}{l}\text { Gait, Funct Reach, Pegboard, UPDRS, } \\
\text { BergBalance }\end{array}$} & $1^{\text {st }} 2^{\text {nd }} \ldots . .12^{\text {th }}$ & $\begin{array}{l}\text { Gait, Funct Reach,Pegboard, UPDRS, } \\
\text { BergBalance }\end{array}$ \\
\hline & $\begin{array}{l}\text { Timed Up and Go, and Functional } \\
\text { Reach: everyday before and after } \\
\text { stimulation } \\
\text { Pegboard, at the } 1 \text { st, } 6 \text { th, and } 12 \text { th } \\
\text { sessions, before and after stimulation } \\
\text { PDQ-39, before the } 1^{\text {st }} \text { and after the } 12^{\text {th }} \\
\text { sessions }\end{array}$ & \\
\hline
\end{tabular}

Evaluation of the Effect of the Programme. Evaluation was carried out twice, two days before beginning and 2 days after the end of the intervention period respectively. PD patients were evaluated during their OFF-periods (>12 hr withholding medication); the tests performed were the following: Gait, Pegboard, Functional Reach (FR), UPDRS, and Berg Balance Test (BBT). In addition the Parkinson's disease questionnaire (PDQ-39) was evaluated also, but during ON periods.

Intra-Session Evaluation. In addition to the comparisons made before and after the programme, we performed a series of intra-session evaluations, to know the acute effect of stimulation, so that a number of tests (see Table 2) were carried out just before and after stimulation. The Timed-up-and-go test (TUG), and the FR were tested every day. Hand dexterity (Pegboard) were performed at the first, sixth and last stimulation session. For intra-session evaluation patients were evaluated during ON-periods.

All evaluations were performed by researchers blind to protocol and group assignment.

Evaluation Tests. Gait: Subjects had to walk along a corridor, turn (U turn), and come back (see Fig. 1a); subjects performed this task three times, with a rest of $2 \mathrm{~min}$. The instruction given was "walk along the corridor as you normally do, turn around the object at the end of the corridor, come back and touch this button on the wall."

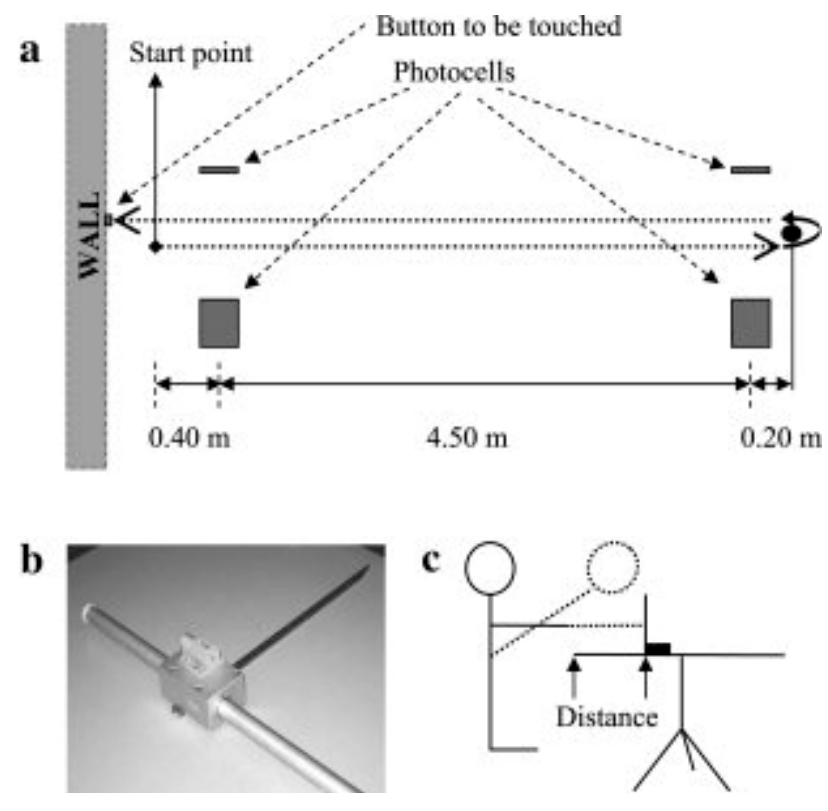

FIG 1. (a) Representation of the task carried out by patients during the gait test, (b) Anthropometer, and (c) Representation of the task performed during the Functional Reach. 
Functional Reach: Subjects had to displace the stick attached to the anthropometer as far as they could, pushing with their fist, without bending the knees or lifting the heels (Fig. 1b,c). Each subject did so three times for each arm, with a rest of $30 \mathrm{~S}$ between trails. The distance between the initial and the final position was taken as the functional reaching distance.

Berg Balance Test: Briefly, the BBT9 is a 14-item scale widely used for assessing balance. The items are scored from 0 to 4 on the basis of the ability to complete a task, the higher the score the more independently performed the tasks.

UPDRS: The total score and the motor section of the scale were taking into account.

Purdue Pegboard Test: This was used to test hand dexterity—subjects had to place as many pegs (small steel rods) as possible, sequentially into a row of holes, within $30 \mathrm{~S}$, and the number of pegs were registered. Subjects performed this task three times for each hand.

PDQ-39: PDQ-39 $9^{10}$ measures quality of life in Parkinsonian patients. It has 39 items to be rated (0-4), the higher the score the poorer quality of life.

Timed up and go test: Briefly, for the TUG, patients are seated on a chair and once commanded, they had to stand up, walk at their preferred walking pattern for $3 \mathrm{~m}$, turn, come back, and sit down on the chair again. ${ }^{11}$ The time taken was recorded with a stopwatch. Patients performed this task three times.

Subjects gave their informed consent to the experimental procedure, conformed to the standards set by the Declaration of Helsinki. Procedure was approved by the University of A Coruña Ethics Committee.

Apparatus. For gait evaluation recording system consisted of a series of footswitches worn as insoles in the shoes. The footswitches were connected to a radio-transmitter attached to the subjects' belt. Data (sampled at $1 \mathrm{KHz}$.) were sent to a receiver unit connected to the computer. This configuration allowed the stride cycle time to be registered. Two photocells, placed $4.50 \mathrm{~m}$ apart, were connected to the recording system.

For the FR test an Harpenden Anthropometer (Holtain Ltd), calibrated to the nearest millimetre, was mounted on a tripod (see Fig. 1b,c), so that the functional reaching distance could be obtain from the difference between the starting and the end positions of the moveable part of the anthropometer.

For WBV stimulation was provided by means of a vibrating platform (Fit Massage, PYC Fitness International Inc), commercially available in various countries. The vibrating platform, configurable in frequency (up to $50 \mathrm{hz}$ with an amplitude of $13 \mathrm{~mm}$, model specifications), thrusts the right and left leg upward alternately (in concordance to previous studies, Ebersbach et al. ${ }^{4}$ ).

Analyzed Variables. For the gait test, a number of kinematic variables were analyzed:

-Velocity, it was calculated from the time consumed to cover the straight part of the task, data obtained from the photocells.

-Cadence, it was calculated from data obtained through footswitches, corresponding to the straight part of the task.

-Step amplitude, derived from the combination of velocity and cadence.

-Turn time, obtained from the successive activation of the photocell at the end of the corridor (before and after the turn).

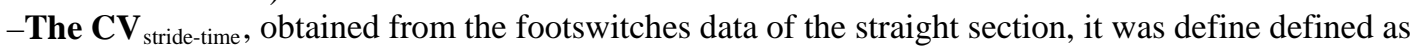

$$
\mathrm{CV}(\%)=(\mathrm{sd} / \mathrm{mean}) \times 100
$$

All the gait variables correspond to the mean of the tree trials.

For the FR test, it was calculated the mean functional reaching distance obtained in the three trials. Scores from both arms were averaged.

The scores from the BBT, PDQ-39 and UPDRS were introduced in the analysis.

The mean number of pegs introduced into the holes in the three trials was the variable analyzed in the Purdue Pegboard test. Scores from both hands were averaged.

The time consumed to complete the test was the variable analyzed in the TUG test (average from the three trials).

\section{Statistical Analysis}

To calculate differences between groups at the pretest a Students " $t$ " for independent samples was applied for each variable. To measure the effect of the whole intervention programme a $2 \times 2$ ANOVA with repeated measures was performed for each of the variables analyzed Preprogramme vs. Postprogramme. The within subjects factor was Evaluation, with two levels (Pretest and Post-test); the 
between subjects factor was the Group (experimental or placebo). To know the intra-session effect a three-way ANOVA with repeated measures was performed. For TUG two within-subjects factor were defined, factor Session with 12 levels (each of the sessions), and factor Pre-Post with 2 levels (intrasession evaluation, prestimulation and poststimulation); the between subjects factor was the Group (experimental or placebo). The same model was applied for the FR. For the Pegboard the model was the same as well, but factor Session had just 3 levels (1st, 6th, and 12th sessions).

Normality of distribution was check by K-S test. Greenhouse-Geisser correction was applied when sphericity was violated. The SPSS package was used for statistical analysis.

\section{RESULTS}

\section{Preprogramme vs. Postprogramme}

\section{Characterization}

Before stimulation groups were equal in all the variables analyzed (see Table 3); for gait variables Students "t" for independent sample yielded no significant differences for any of the kinematics: velocity $(t(19)=0.139 P=0.891)$, cadence $(t(19)=0.034 P=0.974)$, step amplitude $(t(19)=0.233 P=0.819)$, turn time $(t(19)=0.350 P=0.730)$, and $\mathrm{CV}_{\text {stride-time }}(t(19)=0.067 P=0.948)$. Also, the rest of the variables showed no differences between groups: UPDRS $(t(19)=1.466 P=0.159) ; \operatorname{UPDRS}_{\mathrm{III}}(t(19)=$ $1.826 P=0.084)$; BBT $(t(19)=1.030 P=0.316)$; number of pegs $(t(19)=0.557 P=0.584)$; FR distance $(t(19)=0.435 P=0.668)$ and PDQ39 $(t(19)=0.107 P=0.916)$.

TABLE 3. Differences between groups

\begin{tabular}{lll}
\hline & Experimental & Placebo \\
\hline At the preprogramme evaluation & & \\
Gait & & \\
$\quad$ Velocity (m/sc) & $0.747( \pm 0.207)$ & $0.734( \pm 0.212)$ \\
Cadence (steps/sc) & $1.781( \pm 0.166)$ & $1.785( \pm 0.299)$ \\
Step amplitude (m) & $0.417( \pm 0.096)$ & $0.408( \pm 0.083)$ \\
Turn Time (scs) & $2.041( \pm 1.175)$ & $2.234( \pm 1.338)$ \\
CV stride-time (\%) $_{\text {UPDS (score) }}$ & $4.110( \pm 3.909)$ & $4.021( \pm 1.994)$ \\
UPDRSIII (score) & $48.300( \pm 14.712)$ & $58.090( \pm 15.776)$ \\
Berg Balance (score) & $24.800( \pm 7.052)$ & $30.454( \pm 7.118)$ \\
Functional Reach (mm) & $46.200( \pm 5.223)$ & $42.272( \pm 10.955)$ \\
PegBoard (\# rods) & $207.258( \pm 74.701)$ & $221.357( \pm 73.626)$ \\
PDQ-39 (score) & $7.010( \pm 2.378)$ & $7.542( \pm 1.991)$ \\
Before the 1st stimulation session & $49.500( \pm 15.714)$ & $50.545( \pm 27.042)$ \\
TUG (scs) & & $16.1927( \pm 7.599)$ \\
Pegboard (\# rods) & $13.344( \pm 3.155)$ & $7.8336( \pm 2.346)$ \\
F. Reach (mm.) & $7.550( \pm 2.981)$ & $238.4091( \pm 68.654)$ \\
& $235.067( \pm 76.269)$ & \\
\hline
\end{tabular}

No significant differences were yielded in any variable.

\section{Effect of the Stimulation}

A main effect of factor Evaluation was seen in a number of variables, showing significant change between Pre- and Post-test (Table 4). In the gait variables this was seen in the following: velocity $[F(1,19)$ $=59.462 P \leq 0.001]$; step amplitude $[F(1,19)=34.277 P \leq 0.001]$ and cadence $[F(1,19)=21.746 P \leq$ $0.001]$. Conversely no main effect of factor Evaluation was seen in the $\mathrm{CV}_{\text {stride-time }} F(1,19)=1.623 P=$ 0.218 ; or the turn time $F(1,19)=1.847 P=0.190$.

For the rest of the tests, the effect of the programme led FR to increase $[F(1,19)=65.229 P \leq 0.001]$; as well as to increase the number of pegs in the Pegboard test $[F(1,19)=25.617 P \leq 0.001]$; to reduce the total UPDRS score and the UPDRS III $_{\text {score }}[F(1,19)=32.903 P \leq 0.001$ and $F(1,19)=11.929 P=0.003$ respectively) and to reduce the $\operatorname{BBT}$ score $(F(1,19)=22.052 P \leq 0.001)$. PDQ-39 was not affected by the treatment $(F(1,19)=2.332 P=0.143)$. 
TABLE 4. Effect of the stimulation programme

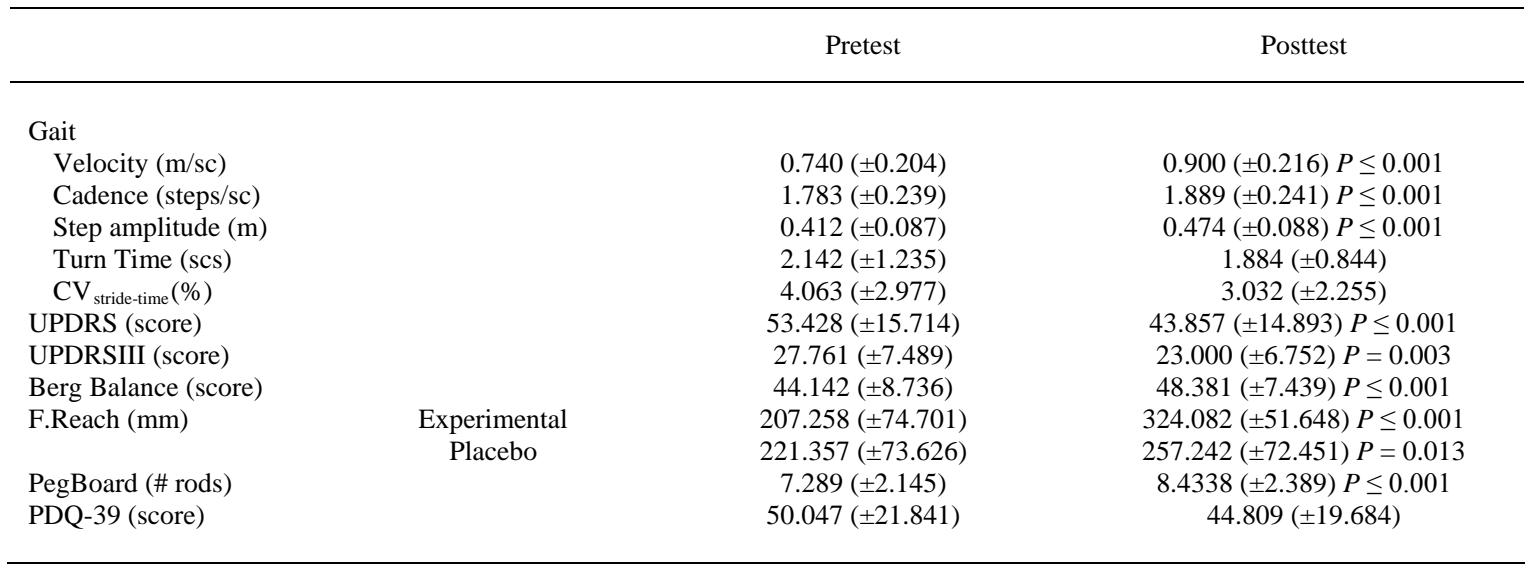

For the rest of the tests, the effect of the programme led FR to increase $[F(1,19)=65.229 P \leq 0.001]$; as well as to increase the number of pegs in the Pegboard test $[F(1,19)=25.617 P \leq 0.001]$; to reduce the total UPDRS score and the UPDRS III $_{\text {score }}[F(1,19)=32.903 P \leq 0.001$ and $F(1,19)=11.929 P=0.003$ respectively) and to reduce the BBT score $(F(1,19)=22.052 P \leq 0.001)$. PDQ-39 was not affected by the treatment $(F(1,19)=2.332 P=0.143)$.

However, the main outcome of this study is provided by the interaction Evaluation*Group-at this level no significant interaction was seen, showing the effect of treatment affected both groups equally. This occurred in all but one of the variables analyzed: velocity $(F(1,19)=1.648 P=0.215$; cadence $(F(1,19)=0.220 P=0.644)$; amplitude $(F(1,19)=1.447 P=0.244) ; \mathrm{CV}_{\text {stride-time }}(F(1,19)=0.421 P=$ $0.524)$; turn time $(F(1,19)=0.039 P=0.846)$; $\operatorname{BBT}(F(1,19)=2.387 P=0.139)$; PDQ39 $(F(1,19)=$ $2.731 P=0.115)$; Pegboard $(F(1,19)=1.792 P=0.196)$; UPDRS and UPDRS ${ }_{\text {III }}(F(1,19)=0.045 P=$ 0.834 and $F(1,19)=0.662 P=0.426$ respectively). The only variable differentially affected by treatment was the FR distance, where a significant interaction was seen $(F(1,19)=18.324 P \leq 0.001)$; however post hoc analysis showed that both groups, experimental and placebo, had increased $(P \leq 0.001$ and $P=0.013$ respectively).

No main effect for factor Group was seen at any variable; $F(1,19)=0.189 P=0.669$ for velocity; $F(1,19)=0.005 P=0.947$ for cadence; $F(1,19)=0.339 P=0.567$ for step amplitude; $F(1,19)=0.486 P$ $=0.494$ for the $\mathrm{CV}_{\text {stride-time }} ; F(1,19)=0.129 P=0.723$ for turn time; $F(1,19)=0.544 P=0.470$ for BBT; $F(1,19)=0.712 P=0.409$ for PDQ39; $F(1,19)=0.052 P=0.822$ for the Pegboard; $F(1,19)=2.247 P=$ 0.150 and $F(1,19)=2.867 P=0.107$ for the UPDRS and UPDRS ${ }_{\text {III }}$, and finally $F(1,19)=0.848 P=$ 0.369 for the FR distance.

\section{Intra-session Evaluations}

The analysis of intra-sessions evaluation yielded similar results to the Preprogramme vs. Postprogramme analysis, even although the medication regime was different, OFF during Preprogramme vs. Postprogramme (except for PDQ-39), and ON during intra-sessions.

\section{Characterization}

Before the first stimulation session, both groups were comparable, as no significant differences were seen at any of the variables analyzed when compared by groups.

For TUG $t(19)=1.100 \& P=0.285$; for the Pegboard $t(19)=0.243 \& P=0.810$; and for the FR $t(19)=0.106 \& P=0.917$ (see Table 3 ).

\section{Stimulation}

Stimulation produced interesting results. For the TUG a main effect of factor Session, $F(2.765,52.529)=5.389 \& P=0.003$, showed patients changed their performance during successive days; however, the lack of interaction of Session*Group $(F(2.765,52.529)=1.091 P=0.358)$ showed this 
effect was also seen in both groups, experimental and placebo, ruling out an effect of vibration on motor execution. Similarly, patients performed differently when we compared the execution before and after stimulation as factor Pre-Post showed a main effect $F(1,19)=5.758 \& P=0.027$, but again this was seen in both groups of patients (experimental and placebo) as interaction between them was not significant $(F(1,19)=0.018 P=0.894)$. The rest of the interactions were not significant (Fig. 2).
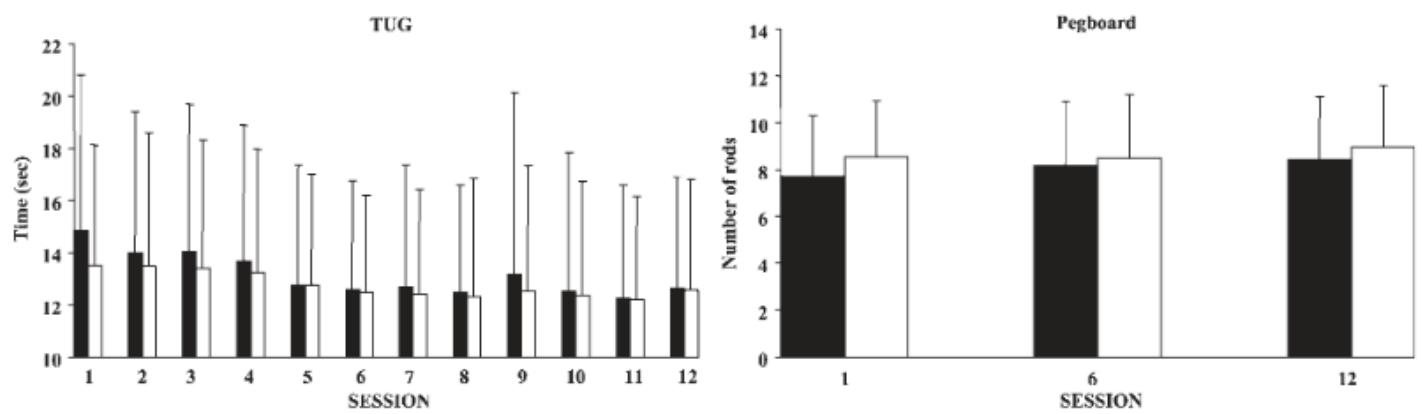

runctional Reach

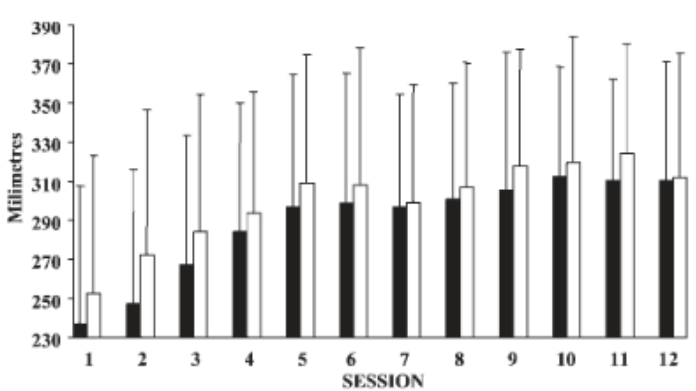

FIG.2. Effect of stimulation on the TUG, Functional Reach, and Pegboard along the different sessions of the programme. Black bars represent evaluation prestimulation, and clear bars represent evaluation poststimulation, both within each session of the programme.

For the Pegboard the only factor to have a main effect was factor Pre-Post $F(1,19)=18.100 P \leq$ 0.001 , however, this again happened for both groups, with the interaction Pre-Post*Group not significant $(F(1,19)=0.807 P=0.380)$ (Fig. 2).

Finally, for the FR, the ANOVA showed the reaching distance was affected by the different sessions (factor Session) $F(4.896,93.027)=23.873 P \leq 0.001$. Also factor Pre-Post had a main effect $F(1,19)=$ $47.727 P \leq 0.001$, proving a different performance before or after the course of stimulation; this was also seen between the different sessions as the interaction Session*Pre-Post was also significant $(F(11,209)=$ $2.058 P=0.025)$. However, the interaction Session*Pre-Post*Group was not significant, proving all changes in performance occurred in both groups $(F(11,209)=1.310 P=0.221)$ (Fig. 2).

Factor group was not significant at any variable; $F(1,19)=0.650 P=0.430$ for TUG; $F(1,19)=0.041$ $P=0.841$ for the Pegboard; and $F(1,19)=0.235 P=0.633$ for the FR.

\section{DISCUSSION}

The main outcome of this study shows that a therapy based on WBV appears to affect Parkinsonian symptoms because of a significant placebo effect. In this study a set of motor tests mainly oriented to evaluate gait and balance, as well as other conditions such as hand dexterity, were equally affected by two protocols which differed only in the application of WBV. This was seen either before vs. after a rehabilitation programme lasting several weeks as well as when analysing the intra-session effect. Very few studies have assessed the effect of WBV on Parkinsonian motor signs-Turbanski et al. ${ }^{2}$ analyzed the effect of a single session of WBV on postural stability assessed in Parkinsonian patients with a modification of the Four Test Balance Scale on a movable and instable platform; these authors also tested a similar stimulation paradigm on tremor and rigidity assessed through UPDRS. ${ }^{3}$ In both cases an improvement in clinical features was described, which was attributed to WBV. In each case these studies included a control group, but the control groups were aware of their control status-in our study, the control group acted as test of the placebo effect-here placebo patients were treated identically to the 
experimental group, but, crucially, during "stimulation" the vibration platform was not switched on. This suggests that the "effect" of vibration, in a whole battery of clinical tests, using the same stimulation frequency and the same protocol (number of set and duration of each set), ${ }^{2,3}$ either comparing intrasession evaluations as the whole therapy, was no different to a placebo. This then suggests that the strong placebo effect seen in our study might also be responsible of results reported by Turbansky et al. ${ }^{2}$ and Hass et al. ${ }^{3}$ A point of difference, however, between our study and those of Turbansky et al. ${ }^{2}$ and Hass et $\mathrm{al}^{3}{ }^{3}$ is the nature of the stimulus-we used nonstochastic vibration, whereas they used stochastically derived vibration. However, Ebersbach et al. ${ }^{4}$ reported results in line with Turbansky et al. ${ }^{2}$ and Hass et al., ${ }^{3}$ using nonstochastic vibration, just as we used in our study. Interestingly, Ebersbach et al. ${ }^{4}$ reported on the suitability of a therapy based on WBV for several days to improve gait and balance in a similar manner to other rehabilitative strategies, but here, again, the lack of control for placebo effect left a question open. Our study shows similar effects than those reported by Ebersbach et al. ${ }^{4}$ and it clearly seems to be due to a placebo effect.

Our results come from a protocol where PD patients were evaluated during ON and OFF periods, and in both situations results followed the same pattern, so that it seems this effect (placebo) does not depend on the regime of medication. Of course there are more variables to be taken into account, (for instance the stimulation frequency or the number of sessions), but our results seem to show that vibration has not an effect "per se," as the same results were seen without vibration.

The repeated measures analysis of variance model used in this study is appropriate to the dataset and, overall, the approach employed is valid with adequate groups sizes (10 and 11 for experimental and placebo respectively) for pre post analysis. However the sample size for the inter-session analyses is limited with a strong drop in power. This represents a limitation of the study and it is due to the relative lack of increasing power with repeated measures above 7 observations (see for instance Rochon ${ }^{12}$ and Vickers ${ }^{13}$ ). However, we like to point out that this happens only in 2 of 14 variables analyzed (TUG and FR during ON dose, which were evaluated everyday), which, on the other hand, followed the same pattern of change than all the others.

In conclusion, we suggest that future studies regarding WBV should consider the possibility of a placebo effect in the design of paradigms involving Parkinsonian patients.

Acknowledgements. Supported by Xunta de Galicia (Consellería de Educación-2007/000140-0 and Dirección Xeral de I+D+i; PGIDIT06PXIC137004PN), Spain. We would like to thank Dr K.L. Grieve for his comments and corrections on the manuscript, and Professor R. Cao for his comments and advice regarding the statistical section.

Author roles: J.C., J.V., M.C. and P.A. designed research; J.V., M.C. and P.A. performed research; J.C. and P.A. designed and implemented statistical analysis; J.C. and P.A. analyzed data; J.C. and P.A. wrote the first draft of the paper; J.C., J.V., M.C. and P.A. reviewed and critiqued the manuscript;

\section{REFERENCES}

1. Nordlund MM,Thorstensson A. Strength training effects of whole-body vibration? Scand J Med Sci Sports 2007; 17: 12-17.

2 Turbanski S,Haas CT,Schmidtbleicher D, et al. Effects of random whole-body vibration on postural control in Parkinson's disease. Res Sports Med 2005; 13: 243-256.

3 Haas CT,Turbanski S,Kessler K,Schmidtbleicher D. The effects of random whole-body-vibration on motor symptoms in Parkinson's disease. NeuroRehabilitation 2006; 21: 29-36.

4 Ebersbach G,Edler D,Kaufhold O,Wissel J. Whole body vibration versus conventional physiotherapy to improve balance and gait in Parkinson's disease. Arch Phys Med Rehabil 2008; 89: 399-403.

5 Haas CT,Buhlmann A,Turbanski S,Schmidtbleicher D. Proprioceptive and sensorimotor performance in Parkinson's disease. Res Sports Med 2006; 14: 273-287.

6 Tinetti ME. Preventing falls in elderly people. N Engl J Med 2003; 348: 42-49.

7 de la Fuente-Fernández R,Ruth TJ,Sossi V,Schulzer M,Calne DB,Stoessl AJ. Expectation and dopamine release: mechanism of the placebo effect in Parkinson's disease. Science 2001; 293: 1164-1166.

8 de la Fuente-Fernández R,Stoessl AJ. The placebo effect in Parkinson's disease. Trends Neurosci 2002; 25: 302306.

9 Berg KO,Wood-Dauphinee SL,Williams JI,Maki B. Measuring balance in the elderly: validation of an instrument. Can J Public Health 1992; 83( Suppl 2): S7-S11

10 Jenkinson C,Fitzpatrick R,Peto V,Greenhall R,Hyman N. The Parkinson's Disease Questionnaire (PDQ-39): development and validation of a Parkinson's disease summary index score. Age Ageing 1997; 26: 353-357. 
11 Podsiadlo D,Richardson S. The timed "Up \& Go": a test of basic functional mobility for frail elderly persons. J Am Geriatr Soc 1991; 39: 142-148.

12 Rochon J. Sample size calcutations for two-group repeated measures experiments. Biometrics 1991; 47: 13831398.

13 Vickers AJ. How many repeated measures in repeated measures designs? Statistical issues for comparative trials. BMC Med Res Methodol 2003 27; 3:22. 